\title{
Editorial Review
}

\section{Radiotherapy in head and neck cancer}

Ionizing radiation has been used as a treatment for head and neck cancer for many years and in many ways. It can be delivered from radioactive sources inserted into, or placed in close proximity to, tumours (brachytherapy), or delivered from a distance (teletherapy), from either a large radioactive source, or in the form of high energy X-rays, or particle beams. For head and neck cancer in the 1990 's, the majority of treatment uses X-rays generated by linear accelerators, many of which can also generate electron beams; these too have a useful role. The techniques of radioactive implants remain an important part of the Radiotherapist's armamentarium in head and neck cancer, the most widely used source these days being Iridium-192. While clinical trials have expended much effort investigating ways of improving results with external beam radiotherapy, scant attention has been paid to comparative evaluation of brachytherapy. Yet this Cinderella among non-surgical modalities can give results in terms of local control, in those centres that continue to use it widely for $T_{1}$ and $T_{2}$ oral cancers, that bear comparison with anything achieved by other means, including surgery (Benk et al., 1990; Pernot et al., 1995).

The indications for using radiotherapy are also various. It can be used to palliate symptoms of advanced disease (although in head and neck cancers its use in this way is much less frequent than in other common tumours), it is used postoperatively to improve local and regional control, it is used in treating clinically uninvolved nodal areas to reduce the risk of relapse at these sites (prophylactic irradiation) and, of course, it can be used as primary therapy given with curative intent. This last area will be the focus of attention here, as it has been the subject of much recent clinical investigation.

Because of their accessibility to direct visual examination and palpation, assessment of response or recurrence and of reactions to treatment are often much easier than with most other tumours, and because of the challenge the disease offers the Radiotherapist, who finds he can cure a substantial portion of patients while others with apparently similar tumours are not cured, squamous carcinomas of the upper aero-digestive tract hold a particular fascination for him. By studying the radiotherapy of head and neck cancer, many lessons have been learnt that have been applicable to radiotherapy in a more general sense, and over a period of decades trials addressing issues of a general interest in radiotherapy have tended to focus on head and neck cancer. The list includes trials with hyperbaric oxygen, radiosensitizers, fast neutrons, and, particularly over recent years, trials addressing the fractionation question and the integration of chemotherapy into radiotherapy schedules. Some exciting results have been emerging.

Long before the dawn of the modern era of megavoltage equipment, it had become internationally accepted practice to deliver radiotherapy on the basis of one treatment per day, five days per week, over a period of some weeks (Thames, 1992). Such schedules made for convenient working practices, and it was only in the 1970's that emerging laboratory data led Radiotherapists to think that perhaps alternative fractionation schedules might sometimes give better results. It has been found that in some tumours (notably squamous carcinomas of the head and neck), the clonogenic cells responsible for continued repopulation multiply quite rapidly, suggesting that prolonged courses of radiotherapy, lasting many weeks, would allow clonogens to continue proliferating and so make up for many of the cells killed by radiotherapy. This would considerably decrease the likelihood of achieving tumour control. (These findings have been considered persuasive enough for the Faculty of Clinical Oncology, Royal College of Radiologists, to produce a document that recommends avoidance of undue prolongation of treatment, and suggests ways of doing so. (Board of Faculty of Clinical Oncology, The Royal College of Radiologists)). The corollary is that shorter overall courses ('acceleration') of treatment might be more effective, as long as this can be done without compromising on the dose of radiation delivered, and this idea needed to be put to the test in clinical trials.

At the same time, evidence was accumulating that in some ways the responses of tumours to radiation differed from those of some normal tissues, and that by breaking the same total dose into a greater number of smaller fractions an equivalent amount of tumour damage could be done, while reducing the late effects on normal tissues. Alternatively an approach of using many, smaller, fractions ('hyperfractionation'), should allow larger total doses to be given, with expected greater anti-tumour effect, (as long as overall treatment times were not prolonged) 
and without resulting in an increase of late normal tissue damage.

Many trials have been conducted to assess whether the theoretical benefits of acceleration and hyperfraction can be translated into clinical gain, and the mid 1990's is seeing an exciting phase during which many of these controlled trials are coming to fruition. In nearly all cases, the two approaches have been combined, this being achieved by treating on more than one occasion each day, and using fraction sizes lower than the 'conventional' size of 2 Gy. In Great Britain, 'CHART' (continuous hyperfractionated accelerated radiotherapy) has been notable among these trials, with a total recruitment of nearly 1000 patients. We are waiting further follow-up before full results of this and of some of the other recently completed trials will be available, but enough data has been collected for some preliminary comments to be made (Horiot et al., 1996; Saunders et al., 1996), and one early trial demands a specific mention. The EORTC radiotherapy group conducted a trial of a 'pure' hyperfractionated regimen and compared it in a randomized trial with conventional radiotherapy, given over the same overall time for tumours of the oropharynx (Horiot et al., 1996). The control arm were treated with a 'standard' dose of $70 \mathrm{~Gy}$, whereas the hyperfractionated treatment delivered $80.5 \mathrm{~Gy}$ in the same overall time, by the expedient of giving two small fractions of $1.15 \mathrm{~Gy}$ on each treatment day. With quite mature follow-up, increased local control was clearly demonstrated with the hyperfractionated schedule, while late morbidity was not increased.

To briefly attempt to summarize a large and complicated literature, it has been shown that modification of fractionation offers potential for improving control of head and neck tumours, and that it is possible to achieve this without paying the price of increased toxicity. No optimum approach has yet been identified, and further trials are ongoing. It is also becoming apparent, perhaps predictably, that individual tumours differ in respect of which schedules are most likely to control them, and we may not be far from developing tests to identify which fractionation schedules are best for which tumours.

Over the years, there has been much debate on what the relative indications are for treatment of primary head and neck tumours with radiotherapy or by surgery. The debate as concerns the more advanced laryngeal cancers (Harwood, 1982; De Santo, 1984), particularly, generated a great deal of heat, but not as much light. To really clarify the issue, what was needed was to perform comparative randomized trials. These have never been adequately conducted (but recently the issue has been indirectly addressed in trials involving chemotherapy - discussed presently). As better local control becomes possible with newer radiotherapy schedules, and perhaps even the possibility of doing this with a reduced incidence of severe post-irradiation effects on normal tissue, the attraction of radiotherapy as an organ-preserving modality is increased. Although it has yet to be firmly established, it is very interesting to note that of the various head and neck sites, laryngeal cancers seem particularly likely to benefit from modified fractionation (Bradley and Morgan, 1991; Medical Research Council 'CHART' Steering Committee). The case for treating moderately advanced laryngeal cancer by primary radiotherapy has been strengthened by these fractionation studies.

The other area of much current interest and promise, that has been explored by good trials, is the integration of chemotherapy with the traditional locoregional treatments of surgery and radiotherapy.

The logistic complexity of combined-modality therapy is compounded by the multitude of available chemotherapeutic agents showing activity in squamous head and neck cancer. No 'ideal' schedule exists, but a statement that a five-day infusion of Fluorouracil, to which Cisplatin is added remains the 'gold standard', would meet little dissent (Al-Sarraf, 1994).

There is a vast body of literature, as might be expected from such a complicated field. Many controlled trials have been performed, although very few have recruited large enough numbers of patients to achieve meaningful conclusions in their own right. Fortunately, rather than attempting to review this literature here we can refer to a recent 'meta-analysis' published by Munro (Munro, 1995). Fifty-four randomized controlled trials were identified, and analyzed together they indicated an absolute survival benefit of 6.5 per cent (confidence limited 3.1 to 9.9 per cent) for the addition of chemotherapy, in whichever of the various schedules, to radiotherapy. In particular, synchronous chemotherapy and radiotherapy appear beneficial. What the analysis did not, and could not, address was the problem that such treatments produce more toxicity than radiotherapy alone, so the counter argument that equal results could be achieved by simply increasing the intensity of radiotherapy cannot be altogether discounted. Even so, it is clear that the time is approaching when chemotherapy will be considered to have an established part in the management of head and neck cancers. What exactly that part is still requires clarification, but one particularly interesting approach demands some consideration.

In the United States a randomized trial conducted by the Veterans Affairs Group (The Department of Veteran Affairs Laryngeal Cancer Study Group, 1991), addressed the issue of organ preservation in advanced laryngeal cancer, by using neo-adjuvant chemotherapy, with radiotherapy subsequently for patients whose tumours responded. The trial compared this approach to a policy of primary surgery with post-operative radiotherapy. Patients who received neo-adjuvant treatment and whose tumours did not show good evidence of response after two cycles of chemotherapy were also treated surgically, as were any recurrences in the conservatively-treated arm. 
It was found that the organ-conserving strategy resulted in no loss of ultimate local control, nor in any survival disadvantage for patients so treated, and with 65 per cent of the patients preserving their larynx. In an era when Oncologists are increasingly interested in the impact of the treatment upon quality of life, the superiority of the organ-conserving treatment over a policy of primary surgery was deemed evident.

More recently, the EORTC has completed a trial of similar design in patients with hypopharyngeal cancer, and with similar results: patients who respond to initial chemotherapy have good prospects of control with subsequent radiotherapy, whilst those who do not can still be salvaged surgically, and again the conservative approach shows equal ultimate local control and survival to that achieved by primary surgery (Lefebvre et al., 1996).

In recent years Oncologists have paid increasing attention to the quality of life of their patients during and after treatment. Curiously, head and neck cancer, where treatment can have such a major impact on quality of life (especially disruption of eating and speaking) has been relatively little studied so far, but it is a field that is beginning to attract a lot of attention. The ability of radiotherapy to cure head and neck cancer while sparing organ function can be seen as a great strength in this context. The fractionation experiments we have discussed have led us to think that improved tumour control can be achieved without added morbidity (and possibly even with some reduction in morbidity), and the neoadjuvant chemotherapy trials point the way in which patients suitable for organ-sparing treatment can be selected from groups with advanced disease. The place of radiotherapy as a curative primary modality for head and neck cancer has thus been consolidated in this era of 'evidence-based medicine'.

There have also been advances in technology that hold promise for the radiotherapy of head and neck cancer. The evidence from the trials we have discussed has reinforced the message that effective radiotherapy requires delivery of an adequate dose of radiation and, for tumour control, the higher the dose the better. Sophisticated tumour imaging can now be combined with advanced engineering to accurately deliver high doses of radiation to irregularly shaped tumours, while minimizing the dose to the surrounding tumour-free tissue. CT and MRI scanning are now routinely in use as part of treatment planning in the field of head and neck cancer, although true three dimensional treatment remains an exciting possibility.

The last decade of the 20th century is an exciting time for those interested in the radiotherapy of head and neck cancer.

\section{References}

Al-Sarraf, M. (1994) Cisplatin combinations in the treatment of head and neck cancer. Seminars on Oncology 21 (Suppl. 12): $28-34$.

Benk, V., Mazeron, J. J., Grimard, L., Crook, J., Haddad, E., Piedbois, P., Calitchi, E., Raynal, M., Martin, M., Le Bougeois, P., Pierquin, B. (1990) Comparison of curietherapy versus external irradiation combined with curietherapy in Stage II squamous carcinomas of the mobile tongue. Radiotherapy and Oncology 18: 339-347.

Board of Faculty of Clinical Oncology, The Royal College of Radiologists (1996) Guidelines for the management of unscheduled interruption or prolongation of a radical course of radiotherapy.

Bradley, P. J., Morgan, D. A. L. (1991) Radiotherapy of advanced laryngeal cancer using three small fractions daily. Otolaryngology-Head and Neck Surgery 104: 838-841.

Department of Veteran Affairs Laryngeal Cancer Study Group. (1991) Induction chemotherapy plus radiation compared with surgery plus irradiation in patients with advanced laryngeal cancer. New England Journal of Medicine 324: 1685-1690.

De Santo, L. W. (1984) $\mathrm{T}_{3}$ glottic cancer: the options and the consequences of the options. Laryngoscope 94: 1311-1315.

Harwood, A. R. (1982) Carcinoma of the larynx. Journal of Otolaryngology 11 (Suppl. 11): 3-21.

Horiot, J. C., Bontemps, P., Begg, A. C., Le Fur, R., Van den Bogaert, W., Bolla, M., N'Guyen, T., Van den Wijngaert, D., Bernier, J., Lusinchi, A., Stuschke, D., Lopez Torrecilla, J., Jancar, B., Collette, L., Van Glabbeke, M., Pierart, M. (1996) Radiothérapie hyperfractionnée et accélérée dans les cancers de la tête et du cou: résultats des essais de l'EORTC et impact sur la pratique clinique. Bulletin $d u$ Cancer/Radiothérapie 83: 314-320.

Lefebvre, J. L., Chevalier, D., Luboinski, B., Kirkpatrick, A., Collette, L., Satmond, T. (1996) Larynx preservation in pyriform sinus cancer: preliminary results of a European Organization for Research and Treatment of Cancer phase II trial. Journal of the National Cancer Institute 88: 890-899.

Medical Research Council 'CHART' Steering Committee (data awaiting publication).

Munro, A. J. (1995) An overview of randomized adjuvant chemotherapy in head and neck cancer. British Journal of Cancer 71: 83-91.

Pernot, M., Hoffsteter, S., Peiffert, D., Luporsi, E., Marchal, C., Kozminski, P., Dartois, D., Bey, P. (1995) Epidermoid carcinomas of the floor of mouth treated by exclusive irradiation: statistical study of a series of 207 cases. Radiotherapy and Oncology 35: 117-185.

Saunders, M. I., Dische, S., Barrett, A., Parmar, M. K. B., Harvey, A., Gibson, D. (1996) Randomized multicentre trials of 'CHART' vs conventional radiotherapy in head and neck and non-small-cell lung cancer: and interim report. British Journal of Cancer 73: 1455-1462.

Thames, H. D. (1992) On the origins of dose fractionation regimens in radiotherapy. Seminars in Radiation Oncology 2: 3-9.

Address for correspondence:

D. A. L. Morgan,

Consultant Clinical Oncologist,

Nottingham City Hospital NHS Trust,

Hucknall Road,

Nottingham NG5 1PB. 